\title{
Erratum to: A Stabilized Mixed Finite Element Method for Elliptic Optimal Control Problems
}

\author{
Hongfei $\mathrm{Fu}^{1}$ - Hongxing Rui ${ }^{2} \cdot \mathrm{Jian} \mathrm{Hou}^{3}$. \\ Haihong $\mathrm{Li}^{1}$
}

Published online: 8 September 2015

(C) Springer Science+Business Media New York 2015

\section{Erratum to: J Sci Comput DOI 10.1007/s10915-015-0050-3}

The authors would like to correct the numerical results of Section 6 published in the original version as follows:

Example 6.1 For the first example, we take the stabilized parameters $\mu=0.2$ and $\delta=0.5$.

Piecewise linear $C^{0}$ element is adopted for the approximation of the flux $\sigma$. The numerical results and main CPU time are presented in Table 1, and numerical plots for the control, the state, and the flux state are shown in Figs. 1, 2, and 3, respectively.

Example 6.2 For the second example, we consider the stabilized parameters $\mu=\delta=0.5$.

Table 2 shows that a first-order convergence is obtained for the control, which is well matched with the theoretical analysis. Figures 4, 5, and 6 show the approximate profiles of the control, the state, and the flux state, respectively, when the lowest order RT element is adopted for the approximation of the flux $\sigma$.

Both numerical examples support the theoretical analysis very well.

The online version of the original article can be found under doi:10.1007/s10915-015-0050-3.

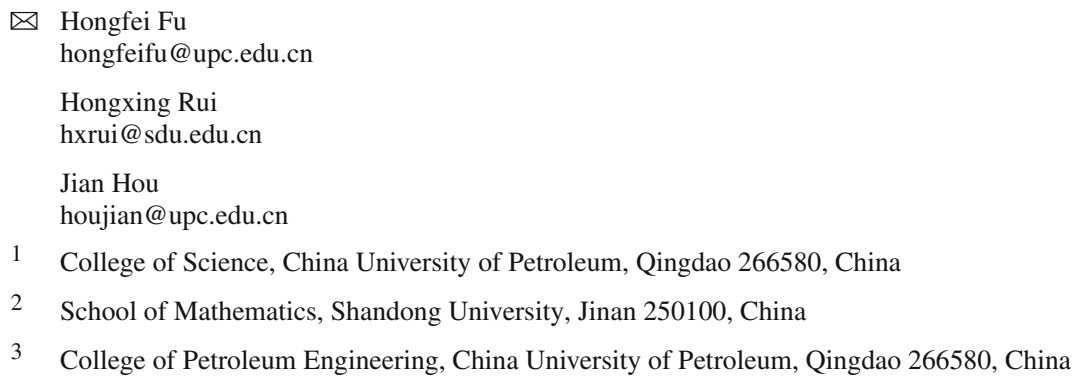


Table 1 Example 1 with piecewise linear $C^{0}$ elements for $\sigma_{h}$

\begin{tabular}{lllll}
\hline$u:$ DoF & $\left\|u-u_{h}\right\|_{L^{2}(\Omega)}$ & $y / \sigma: \mathrm{DoF}$ & \|\|$\left\{y-y_{h}, \sigma-\sigma_{h}\right\}\|\|_{\delta}$ & CPU time (s) \\
\hline 150 & $6.44 \mathrm{E}-02$ & $92 / 92$ & $6.17 \mathrm{E}-01$ & 1.67 \\
602 & $3.22 \mathrm{E}-02$ & $334 / 334$ & $3.07 \mathrm{E}-01$ & 11.13 \\
2390 & $1.54 \mathrm{E}-02$ & $1260 / 1260$ & $1.52 \mathrm{E}-01$ & 76.58 \\
9496 & $7.71 \mathrm{E}-03$ & $4877 / 4877$ & $7.59 \mathrm{E}-02$ & 433.61 \\
\hline
\end{tabular}
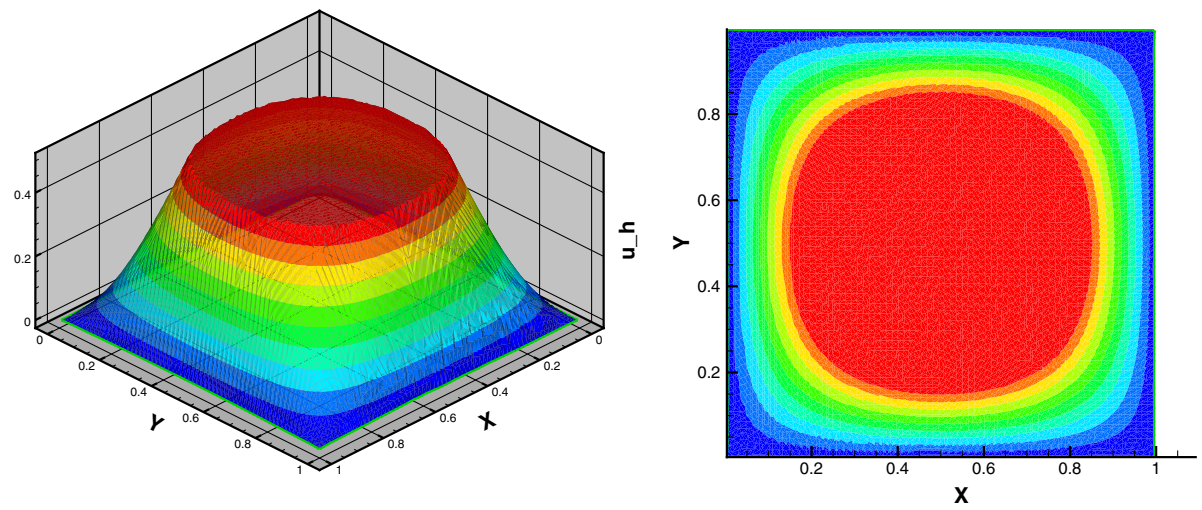

Fig. 1 Approximate control $u_{h}$ (left) and its contour line (right) for Example 1 with $\mu=0.2$ and $\delta=0.5$

Fig. 2 Approximate state $y_{h}$ for Example 1 with $\mu=0.2$ and $\delta=0.5$

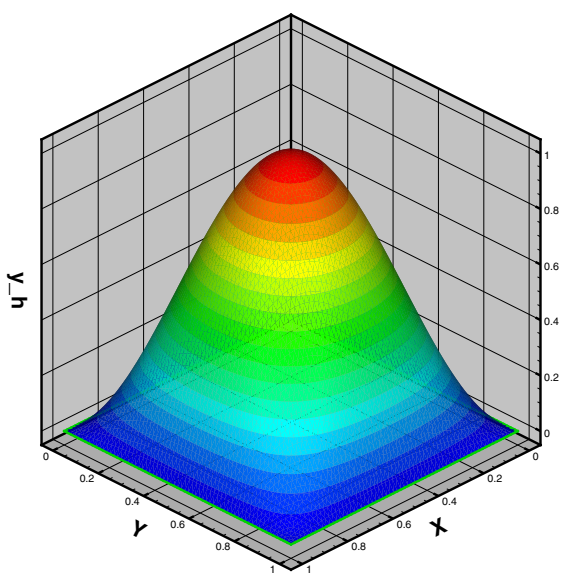



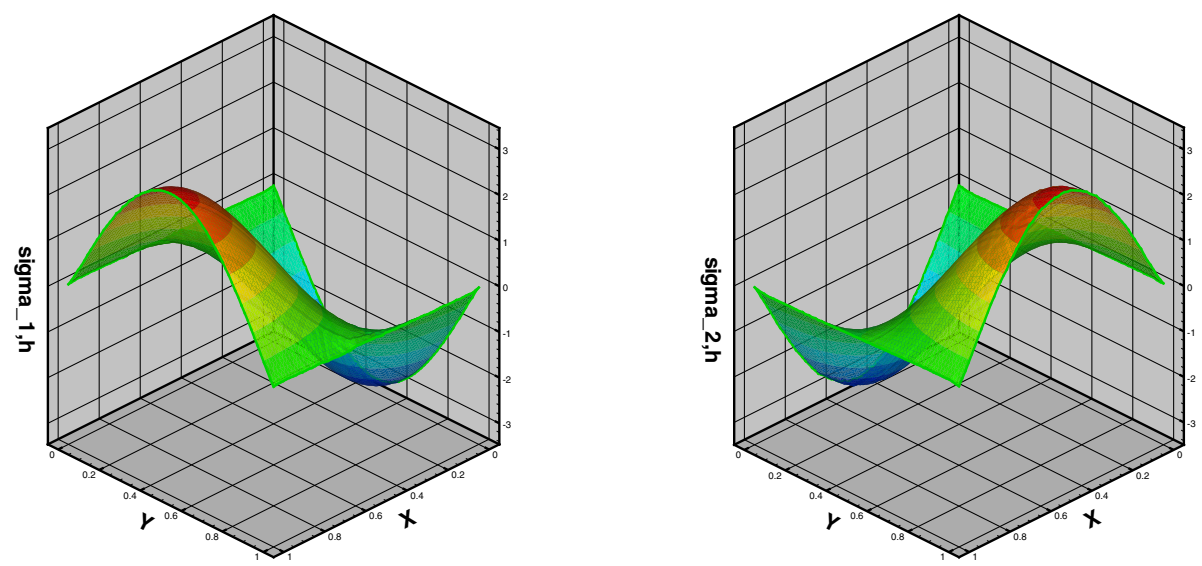

Fig. 3 Approximate flux state $\sigma_{h}$ for Example 1 with $\mu=0.2$ and $\delta=0.5$

Table 2 Example 2 with the lowest order RT elements for $\sigma_{h}$

\begin{tabular}{lllll}
\hline$u:$ DoF & $\left\|u-u_{h}\right\|_{L^{2}(\Omega)}$ & $y / \sigma: \mathrm{DoF}$ & \|\|$\left\{y-y_{h}, \sigma-\sigma_{h}\right\}\|\|_{\delta}$ & CPU time (s) \\
\hline 150 & $5.18 \mathrm{E}-02$ & $92 / 241$ & $3.81 \mathrm{E}-02$ & 2.78 \\
602 & $2.62 \mathrm{E}-02$ & $334 / 935$ & $1.89 \mathrm{E}-02$ & 20.98 \\
2390 & $1.30 \mathrm{E}-02$ & $1260 / 3649$ & $9.47 \mathrm{E}-03$ & 88.34 \\
9496 & $6.52 \mathrm{E}-03$ & $4877 / 14372$ & $4.72 \mathrm{E}-03$ & 469.59 \\
\hline
\end{tabular}
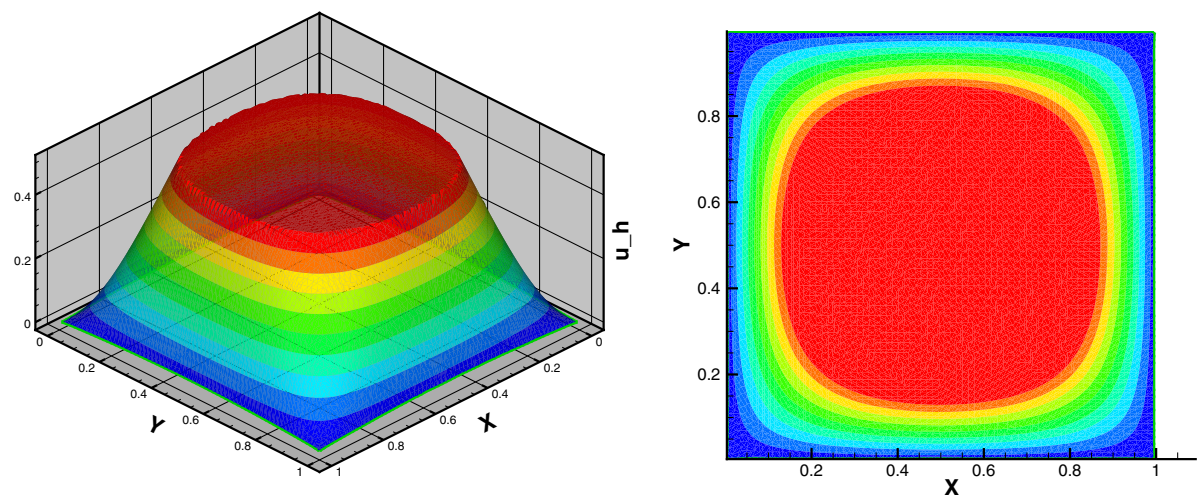

Fig. 4 Approximate control $u_{h}$ (left) and its contour line (right) for Example 2 with $\mu=\delta=0.5$ 
Fig. 5 Approximate state $y_{h}$ for Example 2 with $\mu=\delta=0.5$
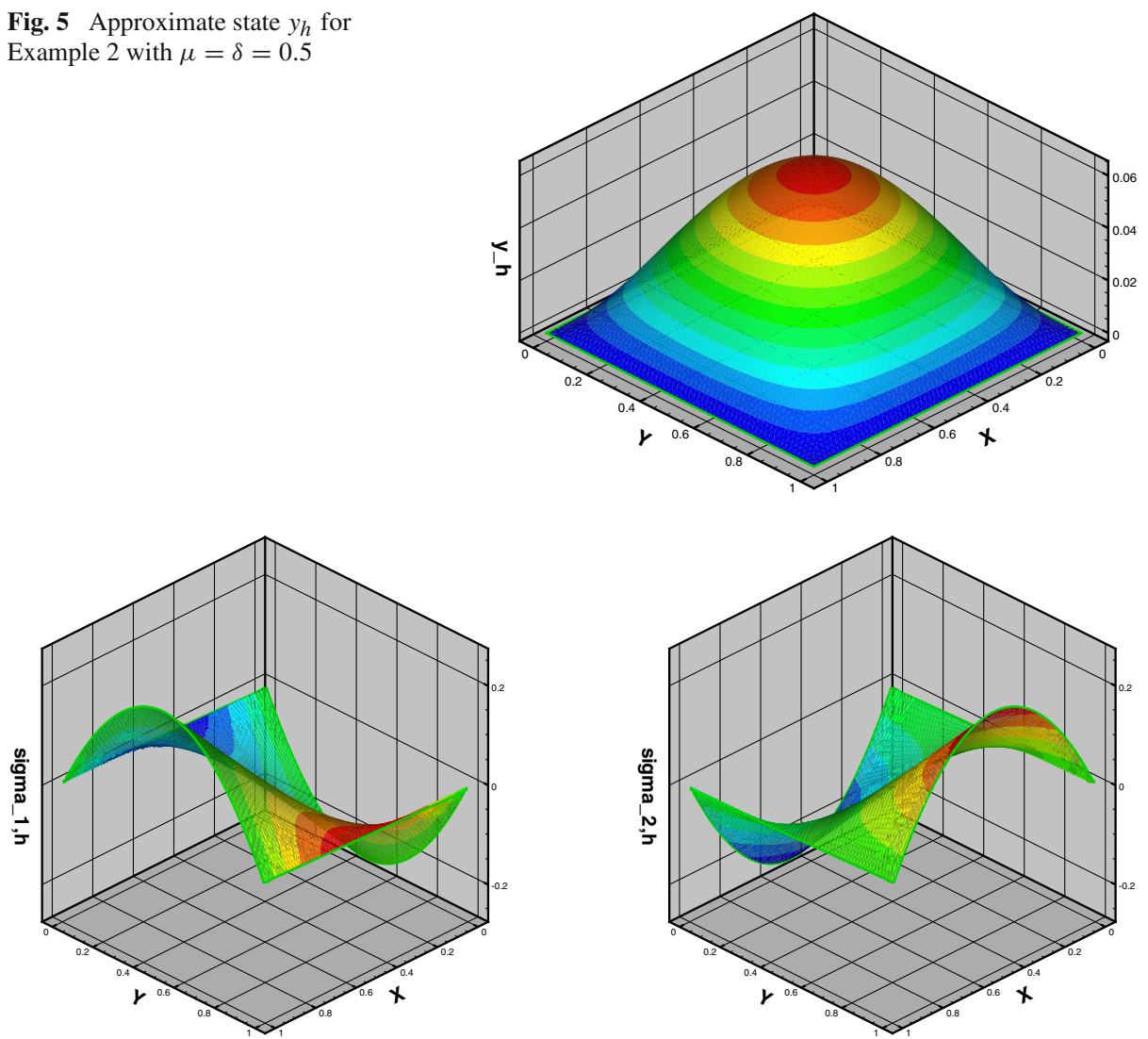

Fig. 6 Approximate flux state $\sigma_{h}$ for Example 2 with $\mu=\delta=0.5$

Acknowledgments The authors would like to thank the editor for giving us a chance to revise the numerical mistakes in the original paper. This work was supported by the National Natural Science Foundation of China (Nos. 11201485, 91330106, and 11171190), the Promotive Research Fund for Excellent Young and Middleaged Scientists of Shandong Province (No. BS2013NJ001), and the Fundamental Research Funds for the Central Universities (Nos. 14CX02217A and 15CX08004A). 